\title{
Changes in Global Trade Patterns in Manufacturing, 2001-2018
}

\author{
Jing Jiang, Linchi Qu \\ School of Economics \& Management, Shanghai Maritime University, Shanghai, China \\ Email: christinejiang2@hotmail.com
}

How to cite this paper: Jiang, J., \& Qu, L. C. (2020). Changes in Global Trade Patterns in Manufacturing, 2001-2018. American Journal of Industrial and Business Management, 10, 876-899. https://doi.org/10.4236/ajibm.2020.105059

Received: April 7, 2020

Accepted: May 5, 2020

Published: May 8, 2020

Copyright $\odot 2020$ by author(s) and Scientific Research Publishing Inc. This work is licensed under the Creative Commons Attribution International License (CC BY 4.0).

http://creativecommons.org/licenses/by/4.0/

\section{(c) (i) Open Access}

\begin{abstract}
This paper used trade data with the year 2001-2018 to construct the global manufacturing multi-layer trade network, analyzed the characteristics of the network and predicted the development trend of the network. The results show that global manufacturing trade has been on the rise and focus on the increase of trade flow; trade be more likely to cooperate with core economic or trade organization; the orientation of returning to manufacturing makes a positive impact on manufacturing trade; network accessibility and compactness are strong, and it tends to be mature. Core-periphery analysis reveals that the United States and China will be the core countries of high, medium and low technology products. The trade forecast results show that the United States and China will conduct fierce manufacturing competition, and the world will form three manufacturing trade dominant regions of North American three countries, Asia-pacific cluster and European cluster.
\end{abstract}

\section{Keywords}

Manufacturing, Complex Networks, Multi-Layer Network, Trade Patterns, Trade Forecast

\section{Introduction}

To manufacturing as the core foundation of the real economy is to maintain national competitiveness and the healthy development of the economy, has become the consensus of the world. Especially after the 2008 global financial crisis, the importance of the real economy was re-cognition, a new round of technological and industrial revolution was on the rise, competition pattern and development trend of manufacturing industry were facing a major adjustment (IMF, 2019). Manufacturing trade is currently the most representative and can reflect changes in the current international trade pattern. How to track the evolution of manu- 
facturing trade and a clearer understanding of the status of various trading countries in the overall trade pattern is an important aspect of the current empirical study of returning to manufacturing.

The international trade network is complex and consists of more than 200 countries and regions around the world and the trade relations between them. Therefore, it is difficult to adopt local national or regional research methods to explain the global trade pattern. The complex network analysis method that emerged at the end of the 20th century reveals the mechanism and evolution of international trade from a global perspective, and explores the interaction mode of trade between countries or regions with its influence on system structure and function. Complex networks are a kind of perspective and method for studying complex systems. Paying attention to the topological structure of individual interactions in the system is the basis for understanding the nature and function of complex systems.

In this paper, quantitative research on 18 years of global trade in manufacturing goods to reveal its network features with complex network analysis. Analyzing the topology of the network, describing the formation and evolution process of manufacturing trade network, reflecting the changes in the status of countries or regions in the process of manufacturing trade transfer, and making a prediction of short-term manufacturing trade. Simultaneously, this study is also a practice of applying complex network analysis methods at the manufacturing trade level. It analyzes the development process of the manufacturing trade pattern over the past 18 years and helps people to understand the development trends in the past 18 years, and provides assistance for other industries that are still in the process of globalization.

Hence, the aim of this paper is to examine changes in trade patterns of manufacturing. The rest of the paper is organized as follows. Section 2 presents the methodology and data. Section 3 introduces the overview of manufacturing trade. Section 4 searches for Manufacturing trade characteristics. Section 5 explores the relationship between manufacturing trading economies. Section 6 forecasts the future development of manufacturing trade. Section 7 finishes with the conclusions.

\section{Methods and Data}

Over the past 15 years, complex network research has developed rapidly. Scholars in various fields have proposed many definitions, methods and tools to find the basic principles and dynamic models of complex networks (Zhang et al., 2017). The first phase mainly revolves around a single static network, including emerging concepts, small-world property (Watts \& Strogatz, 1998), scale-free behavior (Barabasi \& Albert, 1999), community structure (Girvan \& Newman, 2001) etc. In the second stage, in order to understand complex networks, analysis and modeling of temporary empirical networks, such as scientific collaborative networks (Barabasi et al., 2002), biological, Internet, etc., analyze the orga- 
nizational mechanism of network evolution. The third phase of multi-layer complex network applications has attracted wide attention, including the network's centrality, clustering and reciprocity (Federico, Vincenzo, \& Vito, 2014; Gemmetto, Squartini, Picciolo, Ruzzenenti, \& Garlaschelli, 2015; Halu, Mukherjee, \& Bianconi, 2015; Solé-Ribalta, Domenico, Gómez, \& Arenas, 2016).

As a hot spot of emerging research, complex networks have been widely used in international trade by global scholars. The international trade network is a complex system consisting of a set of nodes composed of countries (regions) and a set of trade relations. According to the nature of the network, the international trade network can be divided into different expressions such as unweighted network, weighted network, directed network and undirected network. Some scholars have used complex networks to study bilateral commodity flows between 186 countries (Tzekina, Danthi, \& Rockmore, 2008). Xiaohang Zhang compared the differences in commodity levels and focuses on key countries and regions to analyze typical features in different matrices (Zhang et al., 2017). In an aspect of Topological properties, Squartini et al. (Squartini, Fagiolo, \& Garlaschelli, 2011) use the maximum-likelihood estimation of maximum-entropy models to obtain the results that network descriptions of trade can be significantly simplified by considering the degree sequence only. Barigozzi et al. (Matteo, Giorgio, \& Diego, 2010) show that link-weight distributions of commodity-specific networks are extremely heterogeneous and log normality of aggregate link-weight distribution is generated as a sheer outcome of aggregation. Commodity-specific networks also display average connectivity, clustering, and centrality levels very different from their aggregate counterpart. Shi et al. (Shi et al., 2014) apply the flow analysis method developed in ecology to trading flow networks of different products. They pointed out that the flow networks of products with higher added values and complexity such as machinery and transport equipment have larger exponents, meaning that their trade flow networks are more hierarchical. Assaf Almog (Almog, Squartini, \& Garlaschelli, 2015) tries to make an important step forward in the unification of those two frameworks, by proposing a new gross domestic product (GDP) driven model which can simultaneously reproduce the binary and the weighted properties of the international trade network.

With the research of network science theory, scholars began to use the edge as a breakthrough point to study the connection between systems. A multi-layer network is a network set consisting of multiple single-vertex networks. Each single-vertex network corresponds to one network layer. The network-connected edges contain interconnected edges in the network layer and also contain different layers (Boccaletti et al., 2014; Kivelä et al., 2014) (Figure 1). In recent years, scholars have begun to build a multi-layer network model of international trade, and then study the impact of the coupling between different systems on the international trade system (Lee \& Goh, 2016). Saracco et al. built a 96-layer international merchandise trade network and proposed a multiplicity and multi-reciprocity indicator to measure the relationship between different layers of 


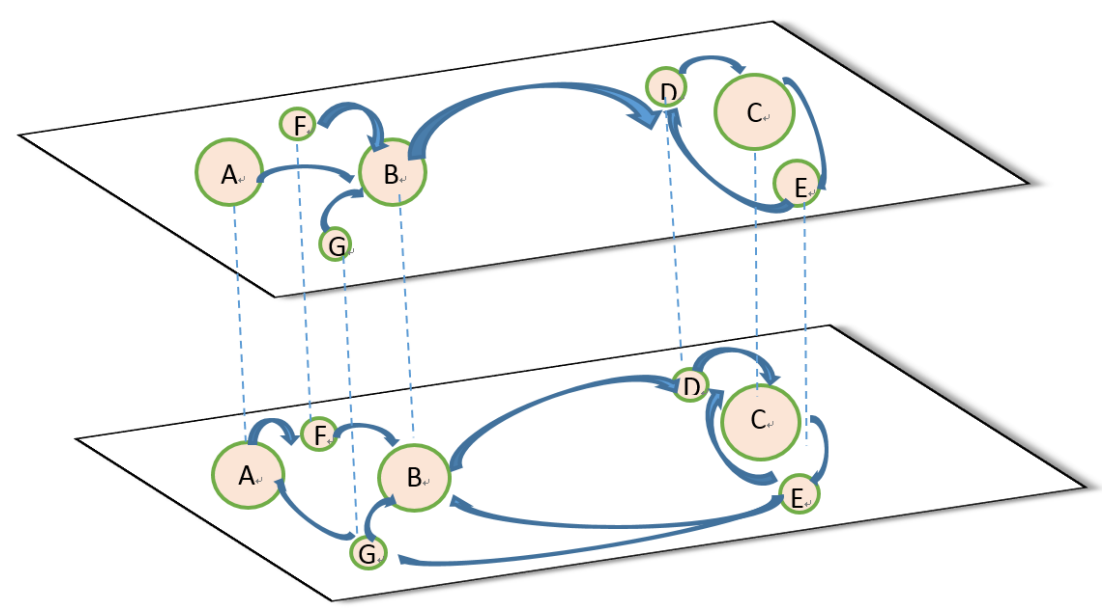

Figure 1. Multi-layer trade network model diagram.

networks in this multi-layer network (Saracco, Clemente, Gabrielli, \& Squartini, 2015). In general, research on the maturity of the manufacturing trade network is still limited.

There are several differences between multi-layer complex networks and ordinary complex networks: One is that the properties of nodes in the same layer network are the same, and the evolution rules are consistent; The other is that the nature of the nodes in different layers of the network is different, which presents another evolutionary rule; There are also evolution rules between different layers of networks. Each evolution rule has different characteristics, and the evolution rule in the same layer is equivalent to a single-layer complex network; different layers of evolution rules are different due to the nature of the nodes; the third evolutionary rule involves the relationship between related sub-networks that promote each other or weaken each other.

\subsection{Construction of Multi-Layer Complex Network}

Multi-layer complex network is essentially a network set composed of multiple (directed or undirected, weighted or unweight) single-layer networks. It is difficult for a single complex network to describe the interrelationship between different trade products in different countries. The purpose of this paper is to reveal the evolutionary trend of international trade relations and trade volume over 18 years. In this study, the transition from single-layer to multi-layer networks, based on a multi-layer network model of trade as the main research method. Use mathematical formulas to show it is $G=(V, E, C)$ (De Domenico et al., 2014), $G$ stands for multi-layer network, here assumes $G$ total $M$ layer, $V=\left\{V_{1}, \cdots, V_{m}\right\}$ represents a collection of nodes in each layer of the network, $E=\left\{E_{1}, \cdots, E_{m}\right\}$ is the collection of contiguous edges in all individual network layers, $E_{a}$ indicates that all nodes are from the set of connected edges formed by the nodes in $V_{a}, E_{a}=\left\{\left(v_{i}, v_{j}\right): i \neq j, v_{i}, v_{j} \in V\right\}$. The network structure of the single-layer network $\alpha$ can be expressed as $\alpha=\left(V_{\alpha}, E_{\alpha}\right)$. $C=\left\{E_{\alpha \beta} \subseteq V_{\alpha} \times V_{\beta} ; \alpha, \beta \in\{1, \cdots, M\}, \alpha \neq \beta\right\}$ is also a collection of side edges 
from two different network layers. $E_{\alpha}$ means intra-layer relationships, $E_{\alpha \beta}$ represents the relationship between layers, When a multi-layer network with multiple subsystems is represented by $G$, the single-layer network $\alpha$ is an abstraction of a certain subsystem.

In practical applications, each layer of a multi-layer complex network model can represent one or a type of product. The solid line connection in the layer also needs to consider the direction and weight of trade, that is, the direction is the direction of trade import and export, and the weight can be traded volume. Unweight networks cannot consider the size difference of trade flows, and can only simply describe the existence or non-existence of trade relations between countries in the network. However, the scale of the weights in the actual international trade system is very different, and there are trade relations with different strengths and weaknesses. The binary representation method of non-zero or 1 cannot accurately describe the characteristics of the international trade system. The weighted network analysis can be used to identify the strength of trade relations, based on the weight network architecture, combined with the network topology, the internal mechanism and evolution characteristics of the international trade network can be more clearly analyzed.

From the above, this study intends to construct a weighted multi-layer manufacturing trade network. The nodes in the network represent the countries or regions involved in the trade. The connection between the nodes indicates that there is a corresponding commodity trade relationship between the corresponding pairs of nodes. The direction of the connected side reflects the trade direction, and the outgoing edge of the node indicates the export trade of the node, the entry into the border is import trade. Correspondingly, the weight of the outgoing or incoming edge symbolizes the specific import and export trade volume of the node and related nodes.

\subsection{Data}

According to the 2001-2018 manufacturing trade statistics, the top 50 countries or regions with the arithmetic mean are selected as research objects. The manufacturing trade of these 50 countries accounts for more than $90 \%$ of global manufacturing trade (see Annex 1, Table A1). The manufacturing trade in this paper is mainly for finished products, so we selected three categories in the UNCTAD STAT database, low-tech manufactures, medium-tech manufactures and high-tech manufactures for data collection, including merchandise trade flow data (Table 1). According to Lall (2000), this study is used to classify the technical content of products (Lall, 2000), taking into account the role of technology in product competitive advantage in terms of $\mathrm{R} \& \mathrm{D}$ weight, economies of scale, barriers to entry, and learning effects. The data presents merchandise trade by trading partner and product based on the SITC commodity classification, Revision 3, at the one- and two-digit level, expressed in thousands of United States dollars. 
Table 1. Technological classification.

\begin{tabular}{|c|c|c|c|}
\hline \multirow{3}{*}{$\begin{array}{l}\text { Low-tech } \\
\text { manufactures L }\end{array}$} & $\mathrm{L} 1$ & Textile/fashion cluster & Textile fabrics, clothing, headgear, footwear, leather manufactures, travel goods \\
\hline & $\mathrm{L} 2$ & Other low technology & Pottery, simple metal parts/structures, furniture, jewellery, toys, plastic products \\
\hline & M1 & Automotive products & Passenger vehicles and parts, commercial vehicles, motorcycles and parts \\
\hline \multirow{2}{*}{$\begin{array}{l}\text { Medium-tech } \\
\text { manufactures M }\end{array}$} & M2 & $\begin{array}{l}\text { Medium technology process } \\
\text { industries }\end{array}$ & Synthetic fibres, chemicals and paints, fertilizers, plastics, iron, pipes/tubes \\
\hline & M3 & $\begin{array}{l}\text { Medium technology engineering } \\
\text { industries }\end{array}$ & Engines, motors, industrial machinery, pumps, switchgear, ships, watches \\
\hline \multirow{2}{*}{$\begin{array}{l}\text { High-tech } \\
\text { manufactures } \mathrm{H}\end{array}$} & $\mathrm{H} 1$ & Electronics and electrical products & $\begin{array}{l}\text { Office/data processing/telecommunications equip, TVs, transistors, turbines, } \\
\text { power-generating equipment }\end{array}$ \\
\hline & $\mathrm{H} 2$ & Other high technology & Pharmaceuticals, aerospace, optical/measuring instruments, cameras \\
\hline
\end{tabular}

(Data from web

http://unctadstat.unctad.org/wds/ReportFolders/reportFolders.aspx).

According to whether the products corresponding to the nodes in the multi-layer trade network are the same, the multi-layer international trade network constructed in this paper has two forms: multi-layer manufacturing trade network based on product technology content classification and time-based multi-layer manufacturing trade network. The former, multi-layer network is a portrait of the three major categories of products a year trade relationship, a total of 17 years of trade data, which eventually build a 17-storey complex network. In the latter case, each layer of the network in the network represents the global manufacturing trade of similar products in different years. Therefore, this study finally constructed a total of 17 three-tier trade networks based on product technology content classification and three time-based 17-storey trade networks.

In order to ensure that each layer network has the same number of nodes, this study will take reservation measures for isolated nodes without even edges. A few countries may not have import and export traffic on certain types of commodities in a certain year, and we still keep the corresponding nodes of the country in the single-layer network constructed by such commodities. The missing observations accounted for $4 \%$ of the theoretical observations, well below the $12 \%$ missing values (Roberts, 2004), so the missing values have less effect.

There are four trade flows of data contains the annual import and export trade situation: the export of country A to country $\mathrm{B}$, the import of country A from country B, the export of country B to country A, and import of country B from country A. In theory, the value of export trade from country A to country B should be the same as the value of import trade from country B to country A. Otherwise, the import trade volume from country A to country B should be equal to the export quota of country B to country A. However, in actual international trade, the traders' prices for traded products are inconsistent. Importing countries generally use CIF prices for imported products, while exporting countries use FOB prices for export products. The FOB price includes the cost of 
goods and domestic transportation costs. Compared with the FOB price, the CIF price also includes the cost of ocean transportation and insurance premiums. Therefore, under normal circumstances, the total trade volume of imported products in the importing country will be higher than the total trade volume of the same batch of products exported by the exporting country. To solve this problem, it is necessary to unify the statistical caliber of both parties to the trade. Simultaneously, the import and export data of country B is treated the same. In this way, the import value of country A is in agreement with the export value of country B.

On the basis of processing the weighted directed matrix, the statistical characteristics of the manufacturing trade network are analyzed $\mathrm{R}$ software and Ucinet. The processed trade data is imported into the statistical analysis software $\mathrm{R}$ as a network adjacency matrix, and the complex network package iGraph of $\mathrm{R}$ is used to construct the network. The overall size of the final two types of multi-layer networks is as follows (Table 2, Table 3).

\section{Analysis of the Overall Pattern of Manufacturing Trade}

The quantitative changes in manufacturing trade from 2000 to 2018 of the selected economies were shown in Figure 2 with an upward curve. In terms of overall scale, global manufacturing trade maintained steady growth in 20012008. There was a significant fluctuation in the rise and fall between 2009 and 2014. In 2014, the scale of manufacturing trade reached a peak of 1131.8 trillion US dollars. Between 2017, manufacturing trade slowed down steadily. The declined

Table 2. The 3-tier manufacturing trade network based on product technology content (17 networks).

\begin{tabular}{cccccc}
\hline Year & NO. of nodes & NO. of connections & Year & NO. of nodes & NO. of connections \\
\hline 2001 & 150 & 7079 & 2010 & 150 & 7429 \\
2002 & 150 & 7094 & 2011 & 150 & 7417 \\
2003 & 150 & 7385 & 2012 & 150 & 7426 \\
2004 & 150 & 7401 & 2013 & 150 & 7419 \\
2005 & 150 & 7408 & 2014 & 150 & 7408 \\
2006 & 150 & 7404 & 2015 & 150 & 7417 \\
2007 & 150 & 7425 & 2016 & 150 & 7409 \\
2008 & 150 & 7423 & 2017 & 150 & 7412 \\
2009 & 150 & 7424 & 2018 & 150 & 7426 \\
\hline
\end{tabular}

Table 3. 17-storey international trade network based on time (3 networks).

\begin{tabular}{ccc}
\hline Technical content & NO. of nodes & NO. of connections \\
\hline High-tech & 850 & 41,904 \\
Medium-tech & 850 & 42,092 \\
Low-tech & 850 & 42,084 \\
\hline
\end{tabular}




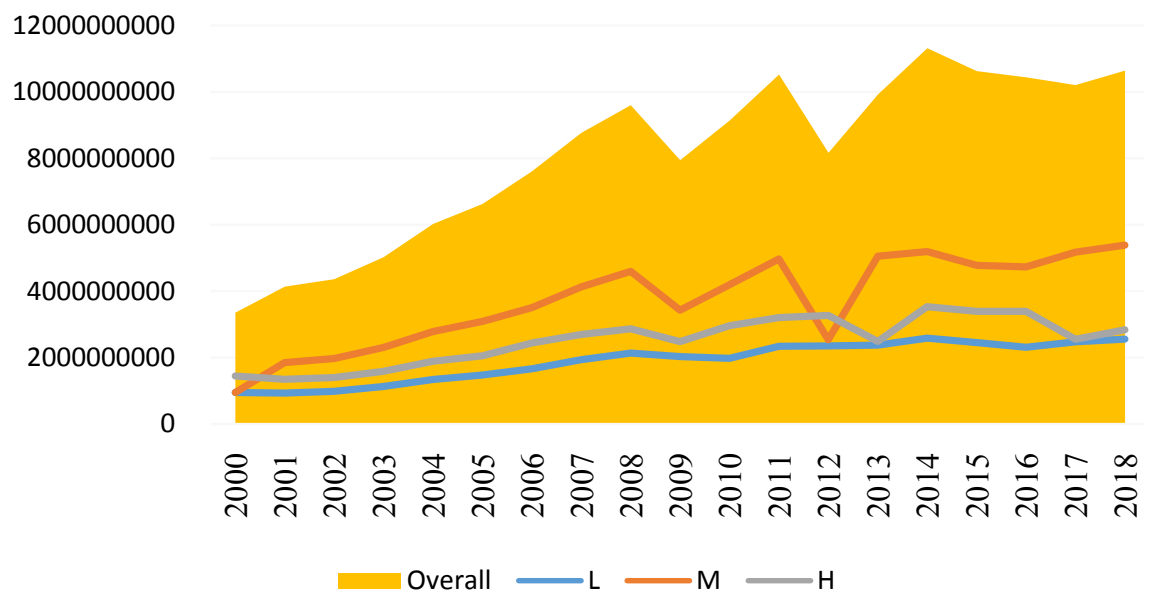

Figure 2. Amount of trade in manufactured goods of different technical content (2001-2018).

trade in 2009 was mainly affected by the global financial crisis, which led to different degrees of international trade. In 2012, due to the gloom of the European debt crisis, the slowdown of the U.S. economy and the prevalence of trade protectionism led to a significant decline in global trade.

In observation of trade structure, low-tech products are mainly composed of necessities and low value goods. Therefore, they are the lowest in the three categories of products. During the period of 2001-2018, they maintained a slow growth and were not significantly affected by external factors. Medium-tech products, as the largest proportion of manufacturing trade, are basically in line with global manufacturing trade changes, but have maintained a gradual upward trend between 2016 and 2017. High-tech products have maintained a slow growth overall, but there has been a small decline in 2013 and 2017, especially in the global trade disputes in 2017. The trade relations between USA and China which the two major trading powers have been ups and downs, which has resulted in significant impact on high-tech products.

\section{Analysis of Statistical Characteristics of Manufacturing Trade Network}

The characteristics of manufacturing trade network can through network distribution, network centrality, network correlation, network clustering identify the imbalanced structure and agglomeration characteristics of global trade, and discuss the status and influence of different economic entities in the global manufacturing trade network. The detection based on network characteristics is a new idea for international trade research decision-making and mining the potential value behind trade big data.

\subsection{Network Degree Distribution}

The degree of a node refers to the number of edges connected to the node in the network, and is a first-order properties. The degree of value in international 
trade indicates the total number of direct trade relations of a country, reflecting the direct trade impact of the country. The larger the value, indicating that the country has more direct trade relations, the greater its influence can reach the node range. For a directed network, each node has an out-degree and in-degree, and the out-degree distribution $P_{\text {out }}(k)$ and the in-degree distribution $P_{\text {in }}(k)$ respectively indicate that a node is taken arbitrarily, and the in-degree value and the out-degree value are $k$ probability.

Scholars have found that the complete random network degree distribution has the characteristics of Poisson distribution, that is, the probability of occurrence of each edge is the same, the degrees of most nodes are basically equal, and close to the network average, far from the peak degree. Another type of network with power-law distribution is called scale-free network, its degree distribution obeys $P(k) \sim k-\gamma, \gamma$ is power law index, usually takes 2 or 3 . Most of the nodes in the network are low in degree, and only a few core nodes (hub nodes) are high degree of value.

In this research, by the strength of this indicator will edge weight combined with even number of edges, a measure of trade in the country for distribution to portray weighted network, helping people learn more about the importance of network degree distribution. The greater of value, the more important a country is in the network. The calculation method of the output strength and the input strength of the network node is as follows:

$$
\begin{aligned}
& S_{i}^{\text {out }}=\sum_{j=1}^{N} \omega_{i j} \\
& S_{i}^{i n}=\sum_{j=1}^{N} \omega_{j i}
\end{aligned}
$$

$\omega_{i j}$ is the weight between node $i$ and node $j$, which is the trade volume between the corresponding countries. The strength of each node corresponds to the trade volume of each country, the total strength of each node is the total network trade volume.

Since the average output strength and average input strength of the global manufacturing trade multi-layer network are equal, and the research methods and research conclusions are similar, the evolution law and distribution of the intensity are represented in this section. Figure 3 gives an evolution curve showing the average annual output strength of the manufacturing trade network based on product technology. The output strength reflects the average export trade volume of countries in the manufacturing trade network. In Figure 3, except for a slight decrease in 2009, the average intensity during the 17 years is generally on the rise. Although the intensity of competition in manufacturing trade continues to increase, with the rise of emerging industries and the maturity of product technology, manufacturing trade is still booming.

Figure 4 shows the Average output strength of international trade networks of three different types of products. $\mathrm{H}, \mathrm{M}$ and $\mathrm{L}$ represent high, medium and low manufactures, respectively. The degree distribution of the three types of products is similar, the degree of the nodes above $60 \%$ is 50 , and only a small part of 


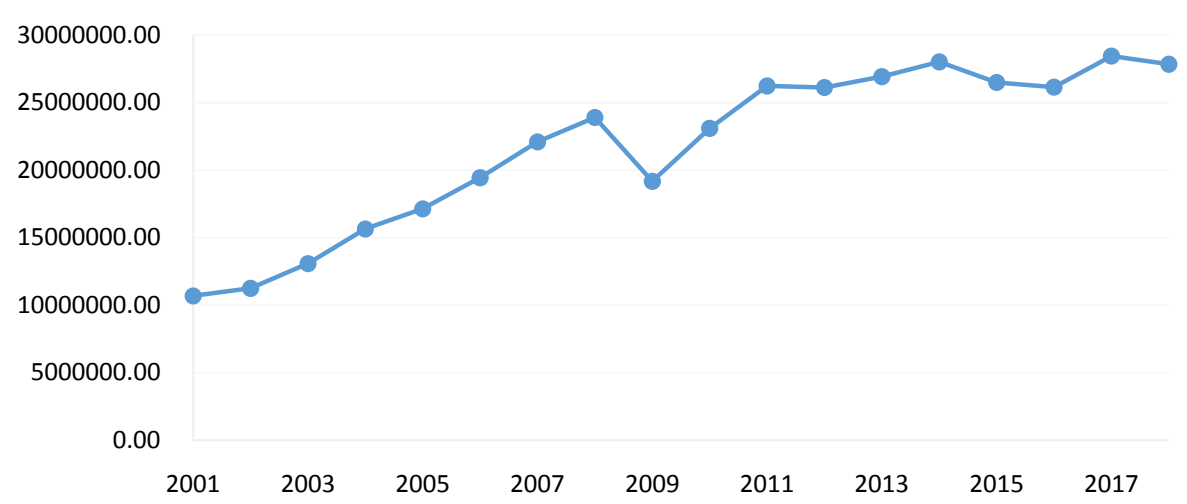

Figure 3. Average output strength curve.

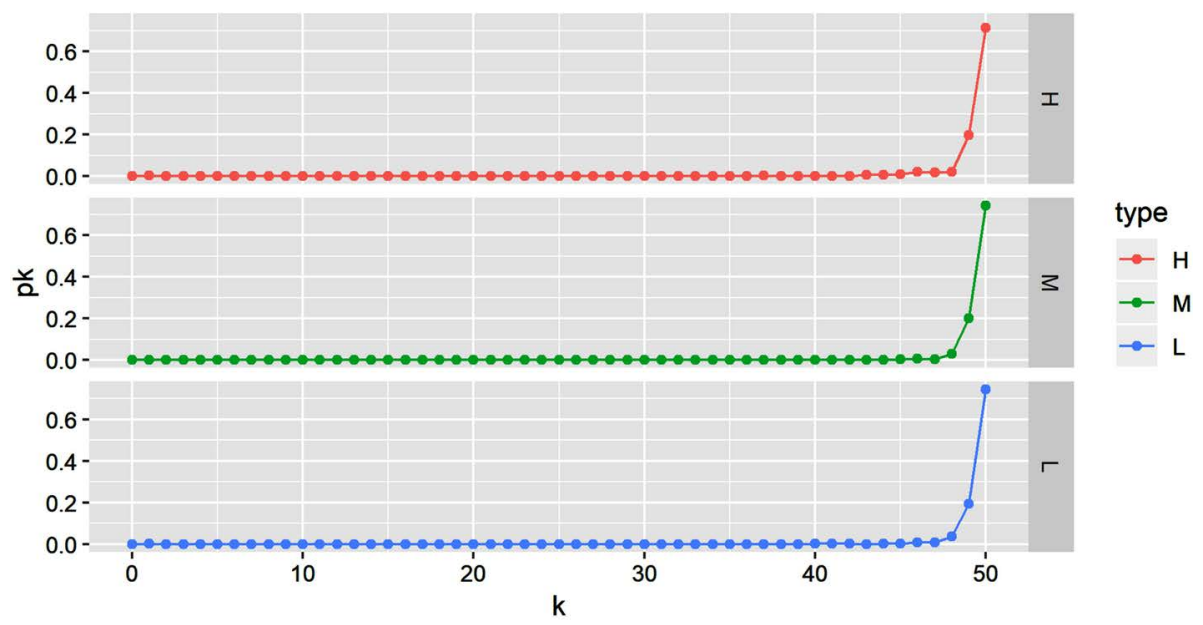

Figure 4. Average output strength of three different types of products.

the node is less than 40. It means that trade network in this study is neither a Poisson distribution nor a scale-free network, but a completely regular network style. There are connections between any two nodes, and the connections are tight, but the trade volume between countries is obviously different. There are trade flows in any two countries in the manufacturing trade network, but the intensity distribution of nodes in the trade network is not random, but there is a great heterogeneity. The key to determining the heterogeneity of node strength is the weight distribution between nodes in the network. In the weight structure of international trade networks, very few countries have most of the trade flows in the network. This reveals that the current focus of the international manufacturing trade system has shifted from establishing trade relations to increasing trade volume.

\subsection{Network Centrality}

The status of the network reflects the influence of the state in the trade network. The centrality is used to measure the quantitative measure of power of the network nodes, including indicators such as Centrality of a Point, Closeness Centrality, and Betweenness Centrality. 


\subsubsection{Closeness Centrality}

Closeness Centrality reflects the location of a node at the center of the network. The more central the country, the shorter its total distance from all other nodes. The larger the value, the larger the proximity of the node, that is, the central position, which is a measure for not accepting the control of others. If a point is short "distance" with all other points in the network, the point is said to have a higher near center. The closeness of a point is the sum of the shortcut distance of the point and all other points. Its expression is (Almog et al., 2015):

$$
\text { Closeness }_{i}=\frac{1}{\sum_{j=1}^{N} d_{i j}}
$$

where $d_{i j}$ is the shortest path length from node $i$ to node $j$. The larger the value close to the center, the point is not the core point of the network.

\subsubsection{Betweenness Centrality}

In contrast to Closeness Centrality, the Betweenness Centrality considers a cost-effective node for spreading to a much larger, more comprehensive network, which represents the importance of the node with the shortest number of paths through the node. When nodes are on many shortest paths, it shows the node's ability to control the flow of resources in the network. The Betweenness Centrality is defined as:

$$
\text { Betweenness }_{i}=\sum_{j}^{n} \sum_{k}^{n} S P_{j k}
$$

where $S P_{j k}$ indicates whether the shortest path between node $j$ and node $k$ passes through node $i$, if passes the node $i S P=1$, otherwise $S P=0$. Betweenness Centrality does not distinguish between in and out. If the Betweenness Centrality is 0 , it means that the point cannot control any trade subject, at the edge of the network. If the Betweenness Centrality is 1 , it is thought that this point can control $100 \%$ of other trade entities, and it has a lot of power at the core of the network. Betweenness Centrality degree does not distinguish between in and out. If the Betweenness Centrality is 0 , it means that the point cannot control any trade subject and is at the edge of the network. If the Betweenness Centrality is 1, it is thought that this point can control $100 \%$ of other trade entities, and it has a lot of power at the core of the network.

With the help of the characteristics of network centrality, the status of global manufacturing trade countries or regions is analyzed. Table 4 shows the top 5 nodes and their attributes of the manufacturing trade network's access the output strength and the input strength, Closeness Centrality and Betweenness Centrality. Measure represents the type of central indicator, and Centralization represents the central indicator score.

From 2013 to 2011, China ranked the top 5 input strength, and the export product types were high-tech products, indicating that China's manufacturing trade volume has benefited from domestic innovation-driven policy guidance in the past five years, and was no longer limited to low-tech products. The focus of the product has shifted to high-tech products and has become an important hub 
Table 4. The top 5 of the manufacturing trade network centrality.

\begin{tabular}{|c|c|c|c|c|}
\hline Measure & Node & Type & Year & Centralization \\
\hline Output strength & China & High-tech & 2013 & $378,860,984.4$ \\
\hline Output strength & China & High-tech & 2014 & $392,745,275.9$ \\
\hline Output strength & China & High-tech & 2015 & $387,971,233.1$ \\
\hline Output strength & China & High-tech & 2016 & $368,827,039$ \\
\hline Output strength & China & High-tech & 2017 & $412,474,878.4$ \\
\hline Intput strength & United States of America & High-tech & 2014 & $249,451,389.7$ \\
\hline Intput strength & United States of America & High-tech & 2015 & $259,142,456.8$ \\
\hline Intput strength & United States of America & High-tech & 2016 & $257,737,313.3$ \\
\hline Intput strength & United States of America & High-tech & 2017 & $277,658,417.6$ \\
\hline Intput strength & United States of America & Medium-tech & 2017 & $244,659,672.8$ \\
\hline Out-closeness centrality & Argentina & High-tech & 2001 & 0.000275398 \\
\hline Out-closeness centrality & Kuwait & High-tech & 2001 & 0.000290576 \\
\hline Out-closeness centrality & Nigeria & High-tech & 2001 & 0.000298566 \\
\hline Out-closeness centrality & Venezuela (Bolivarian Rep. of) & High-tech & 2001 & 0.000277744 \\
\hline Out-closeness centrality & Viet Nam & High-tech & 2001 & 0.000284709 \\
\hline In-closeness centrality & Indonesia & High-tech & 2001 & $4.47 \mathrm{E}-05$ \\
\hline In-closeness centrality & Indonesia & Medium-tech & 2001 & $4.50 \mathrm{E}-05$ \\
\hline In-closeness centrality & Indonesia & Low-tech & 2001 & $4.53 \mathrm{E}-05$ \\
\hline In-closeness centrality & Indonesia & Medium-tech & 2002 & $4.50 \mathrm{E}-05$ \\
\hline In-closeness centrality & Indonesia & Low-tech & 2002 & $4.53 \mathrm{E}-05$ \\
\hline Betweenness Centrality & Venezuela (Bolivarian Rep. of) & Low-tech & 2010 & 4949 \\
\hline Betweenness Centrality & Venezuela (Bolivarian Rep. of) & Low-tech & 2014 & 5270 \\
\hline Betweenness Centrality & Venezuela (Bolivarian Rep. of) & Low-tech & 2015 & 5366 \\
\hline Betweenness Centrality & Venezuela (Bolivarian Rep. of) & Low-tech & 2016 & 5151 \\
\hline Betweenness Centrality & Venezuela (Bolivarian Rep. of) & Low-tech & 2017 & 5236 \\
\hline
\end{tabular}

for the manufacturing trade system. World high-tech manufacturing centers are being gradually shifting eastward. The USA ranked top 5 output strength, as the major exporting countries, is strongly dependent on exports from other countries. Interestingly, in 2014-2016, only high-tech products depended strongly, and import demand for medium-tech products in 2017 also began to soar. Indonesia occupied the top 5 in the period from 2001 to 2002 in aspect of in-closeness centrality, indicating that during this period, Indonesia has import trade flows from many countries, with a high degree of import dependence and a wide range of products. The top five Betweenness Centrality was occupied by Venezuela, which was a relay station for low-tech product trade flows, which was also consistent with the fact that the national production capacity was low, and low-tech products such as textiles and plastics also needed to rely on imports. 
The top five nodes close to out-closeness Centrality were all high-tech products, and the countries were scattered, including Argentina, Tweed, Nigeria, Venezuela (Bolivia) and Vietnam. In terms of technical classification, high-tech products have become the leading force in the development of international trade due to their high technical content, high monopoly and high value, and have received widespread attention from all countries. From the time of trade occurrence, in addition to the Closeness Centrality inter-node trade between 2001 and 2002, other high-central node trades occurred in the past five years, which explained after the financial crisis, the incentive orientation of countries to return to manufacturing has had a relatively positive impact on world manufacturing trade.

\subsection{Network Degree Correlation}

Degree correlation is one of the characteristics of a real network that is, randomly selecting the association of two nodes connected by one edge on the degree value. A random network is the correlation of non-existence, because the characteristic of a random network is to randomly select two nodes that are not connected to connect, so there is no correlation of degrees. However, the reality network is often the relevance of the existence, that is, the connection between the nodes follows a certain law. For example, the nodes whose degree distribution obeys the power law distribution tend to connect to the more moderate nodes. When a node of height tends to connect with nodes of other heights, it is called homogenous mixing; when a node of height tends to connect with other low-degree nodes, it is called non-homogeneous mixing. Vazquez proposes a method for quantifying vertex degree correlation (Vázquez, Pastorsatorras, \& Vespignani, 2002), the average of the nearest neighbors of node $i$ is defined as:

$$
k_{n n}(i)=\left(\sum_{j \in V(i)} k_{j}\right) / k_{i}
$$

$V(i)$ represents the set of neighbor nodes of $i$.

Average the nearest neighbor averages for nodes with degree $K$ :

$$
K_{n n}(K)=\frac{\sum_{k i=K} k_{n n}(i)}{N P(K)}
$$

$N P(K)$ means the number of nodes with degree $K$. If $K_{n n}(K)$ increases as $K$ increases, the node has a tendency to connect to nodes of the same or greater degree, that is, a homogeneous hybrid network. Conversely, if $K_{n n}(K)$ decreases as $K$ increases, nodes with small degrees tend to connect with the hub node as a heterogeneous hybrid network.

Figure 5 shows the degree-correlation curve of the manufacturing trade network during the period 2001-2018. As the network continues to evolve, the scatter points in the graph continue to aggregate, forming a curve that decreases with $K$, indicating the manufacturing trade network in this study. Countries with low node degrees tend to develop trade relations with countries with high node 


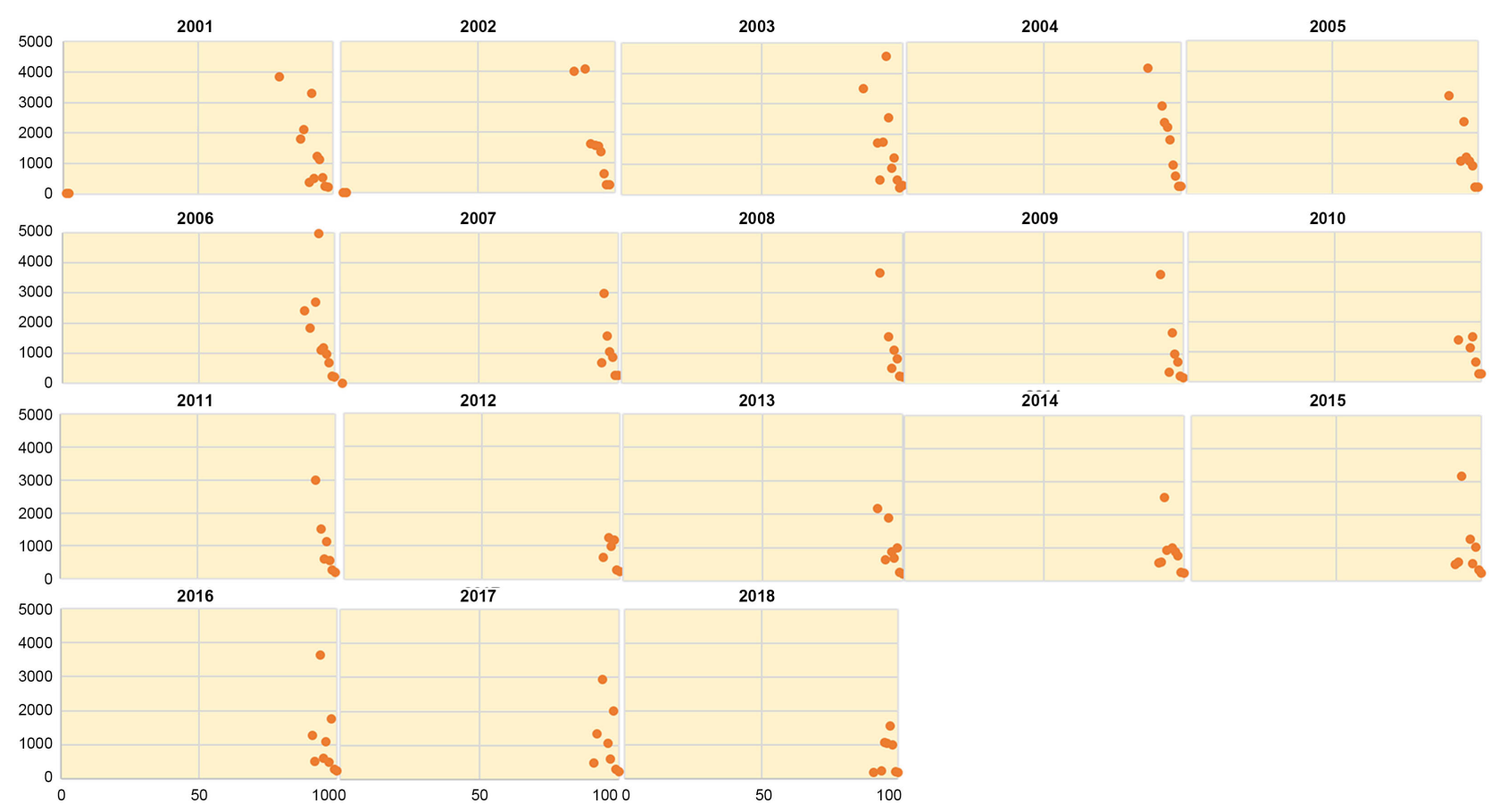

Figure 5. The pictures of manufacturing trade network degree correlation evolution.

degrees. This indicates that due to geographical proximity and cultural similarity, small countries in local areas tend to develop manufacturing trade relations with hub countries in the region, and then form a regional economic cooperation organization with regional powers as the core. Nowadays, the world's largest regional economic organizations include the European Union, APEC, the North American Free Trade Area, and ASEAN. The development of regional economic integration was most remarkable in 2015. Since the US Trump administration took office in 2016, regional economic integration and current isolationism, protectionism, and closures have escalated, and the $K$ point has increased between 2016 and 2018.

\subsection{Clustering Efficient and Average Path Length}

The Clustering Efficient is the probability that there is a trade relationship between trading partners of a country in the network, reflecting the connectivity between the trading partners of the country. If the country's trading partners are closely relative, then the country's Clustering Efficient is higher; on the contrary, if the relationship between a country's trading partners is loose, then the country's clustering coefficient is low. The Clustering Efficient is used to describe the degree of aggregation among nodes in the network. The Clustering Efficient $C_{i}$ of a certain node indicates the ratio of the actually existing connection $N_{i}$ and the most likely connection among all neighbor nodes $k_{i}$ of the node. Only those vertices with at least two neighbors have a practical meaning in calculating the clustering coefficients. The network Clustering Efficient $C$ is the average of the clustering coefficients of all nodes, and is specifically defined as: 


$$
\begin{gathered}
C_{i}=\frac{2 N_{i}}{k_{i}\left(k_{i}-1\right)} \\
C=\frac{\sum_{i=1}^{N} C_{i}}{N}
\end{gathered}
$$

Obviously, $0 \leq C \leq 1$. When $C=0$, it means that all nodes in the network are isolated nodes, that is, there are no connected edges. When $C=1$, it means that any two nodes in the network are directly connected, it means that the network is a completely regular network. If the degree of aggregation of nodes in the network is high, the clustering efficient is large and the connection between nodes is closer.

Distance is a hot issue in the field of international trade, and an important measure of the characteristics of complex network structures. It has an important influence on attributes such as network connectivity. The distance $l_{i j}$ between any two nodes in the network is the number of steps from one of the nodes to the shortest path of the other node. The network average path length $\mathrm{L}$ is defined as the average of the clusters of all nodes in the network, that is,

$$
L=\frac{2}{N(N-1)} \sum_{i=1}^{N-1} \sum_{j=i+1}^{N} l_{i j}
$$

The average clustering efficient of network nodes in the world's manufacturing trade for nearly 17 years is calculated. It is found that the average aggregation coefficient of the network nodes is between 0.9465 and 0.9430 , indicating that there is a close trade relationship among the selected 50 countries or regions. It means highly credible of the research subjects representing global manufacturing trade. It can be seen from Table 5, the average path length shows a downward trend, and the clustering efficient keeps rising slightly from 2001 to 2010, and tends to be stable in 2011-2018. The world manufacturing trade countries or regions between 2001 and 2018 have remained relatively stable, with strong accessibility and closeness, and high efficiency in the transmission of

Table 5. Clustering efficient and average path length of manufacturing trade network.

\begin{tabular}{cccccc}
\hline Year & $\begin{array}{c}\text { Average Path } \\
\text { Length }\end{array}$ & Clustering Efficient & Year & $\begin{array}{c}\text { Average Path } \\
\text { Length }\end{array}$ & Clustering Efficient \\
\hline 2001 & 1.671013883 & 0.943844369 & 2010 & 1.664983 & 0.946839 \\
2002 & 1.66933109 & 0.944938077 & 2011 & 1.666128 & 0.946299 \\
2003 & 1.669427609 & 0.945790851 & 2012 & 1.665522 & 0.946034 \\
2004 & 1.668080808 & 0.946033779 & 2013 & 1.66633 & 0.945758 \\
2005 & 1.667474747 & 0.946033779 & 2014 & 1.66835 & 0.943769 \\
2006 & 1.667138047 & 0.946839142 & 2015 & 1.66734 & 0.943769 \\
2007 & 1.665454545 & 0.946033779 & 2016 & 1.668081 & 0.944036 \\
2008 & 1.665723906 & 0.946574076 & 2017 & 1.667744 & 0.944281 \\
2009 & 1.665656566 & 0.946574076 & 2018 & 1.667823 & 0.944421 \\
\hline
\end{tabular}


commodity trade. With the acceleration of the globalization process, due to the improvement of the multilateral trading system and the strengthening of regional economic exchanges, the regional economic grouping in the manufacturing trade network system has matured.

\section{Core-Periphery Structure Analysis}

If the point of a network is skewed and the point is positively correlated with the point strength, the network has a core-periphery structure (Vázquez et al., 2002). The core-periphery structure analysis is based on the close relationship among the nodes in the network. Considering the nodes in the network into two aspects, namely the core area and the periphery area, the density between the core area members is maximized, and the density between the members of the periphery areas is the smallest. The close relationship between the two areas is not considered, and the core is at the core. The nodes of the area occupy a relatively important position in the network.

This study uses Ucinet software to construct the core-periphery structure of the multi-layer network in manufacturing trade network and divides three types of different products into core members and periphery members. The specific grouping is shown in Table 6. China and the United States are core nodes in the three types of products and are the leaders in today's world manufacturing trade system. In the low-tech product trade network, there are two core countries, and trade occurred in 2014, indicating that 2014 was the peak period of low-tech product trade. The core countries or regions of medium-tech products were distributed in 2017, the old manufacturing powerhouses of Canada, Germany, Japan and emerging manufacturing economies in Mexico. Hong Kong, China has become one of the three representatives of the core areas of high-tech products in 2017. For the time perspective, high-tech and medium-tech products became the core countries' trade targets in 2017, indicating that high-tech and medium-tech

Table 6. Core-periphery structure of three types of products.

\begin{tabular}{ccc}
\hline Product classification & Core node & Year \\
\hline Low-tech & China & 2014 \\
Low-tech & United States of America & 2014 \\
Medium-tech & Canada & 2017 \\
Medium-tech & China & 2017 \\
Medium-tech & Germany & 2017 \\
Medium-tech & Japan & 2017 \\
Medium-tech & Mexico & 2017 \\
Medium-tech & United States of America & 2017 \\
High-tech & China & 2017 \\
High-tech & China, Hong Kong SAR & 2017 \\
High-tech & United States of America & 2017
\end{tabular}


manufacturing have become the focus of strategic competition among economies in the world.

The density matrix of the core-periphery structure is calculated to verify the validity of the model (Table 7). Dividing the density relationship of the entire network into four areas, namely core area, periphery area, core to periphery area, periphery to core area. In this matrix, all data in the core area is 1 , all data in the periphery area is 0 , and data in the non-diagonal area is missing. The correlation between the ideal model matrix and the actual resulting density matrix was calculated by QAP program. The final fits of the three types of products were $0.567,0.537$, and 0.521 , respectively, three of which were statistically significant. This illustrates the model and data fit, which proves that the manufacturing trade network has a core-periphery structure.

\section{Trend of Global Manufacturing Trade}

The international trading system is a typical and evolving nonlinear complex system. The development of trade is affected by various factors such as macroeconomic growth, trade policy changes, technological progress, international environment and natural disasters.etc. The above factors and their non-linear effects are often partly reflected in the evolutionary sequence of international import and export trade, and this evolution cannot be fully accommodated by linear relationships. Making the quantitative forecasting (short-term one year) of three types products in aspects of trade trend and trade flow based on above analysis of the manufacturing trade network (see Annex 2, Table A2, Table A3). The traditional econometric model predicts trade-related problems, it mainly establishes regression models through variables with causality, such as one-dimensional linear model, stepwise regression model, multi-level hierarchical regression model, etc. However, the above methods hardly eliminate the influence of "spurious-regression" between variables. This study selects the HoltWinters algorithm in the time series. HoltWinters. The HoltWinters algorithm belongs to the cubic exponential smoothing model. It considers more influencing factors than the primary/quadratic smoothing exponential model. The degree of fitting is related to the stability of historical data changes. If there are certain rules in historical data changes, the algorithm will often capture the law.

Table 7. Density matrix of core-periphery structure.

\begin{tabular}{cccc}
\hline Product classification & Role & Core & Periphery \\
\hline \multirow{2}{*}{ High-tech } & Core & 0.486 & 0.135 \\
& Periphery & 0.206 & 0.027 \\
\multirow{2}{*}{ Medium-tech } & Core & 0.528 & 0.350 \\
& Periphery & 0.171 & 0.051 \\
\multirow{2}{*}{ Low-tech } & Core & 0.650 & 0.123 \\
& Periphery & 0.277 & 0.077 \\
\hline
\end{tabular}


The HoltWinters function in $\mathrm{R}$ is used to construct a trade forecasting model for three types of products for global manufacturing trade, and to predict and visualize models on the trade data from 2001 to 2018 (Figures 6-8). The short-term

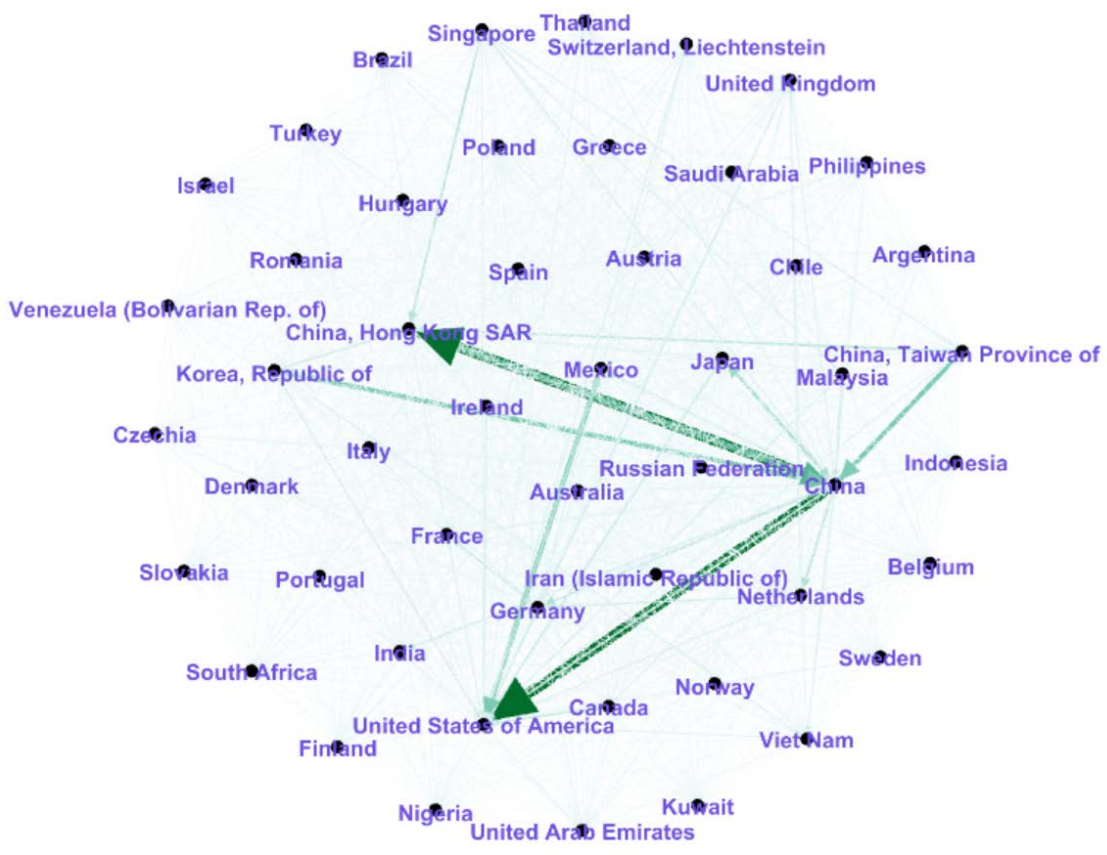

Figure 6. High-tech products trade network (short-term forecast).

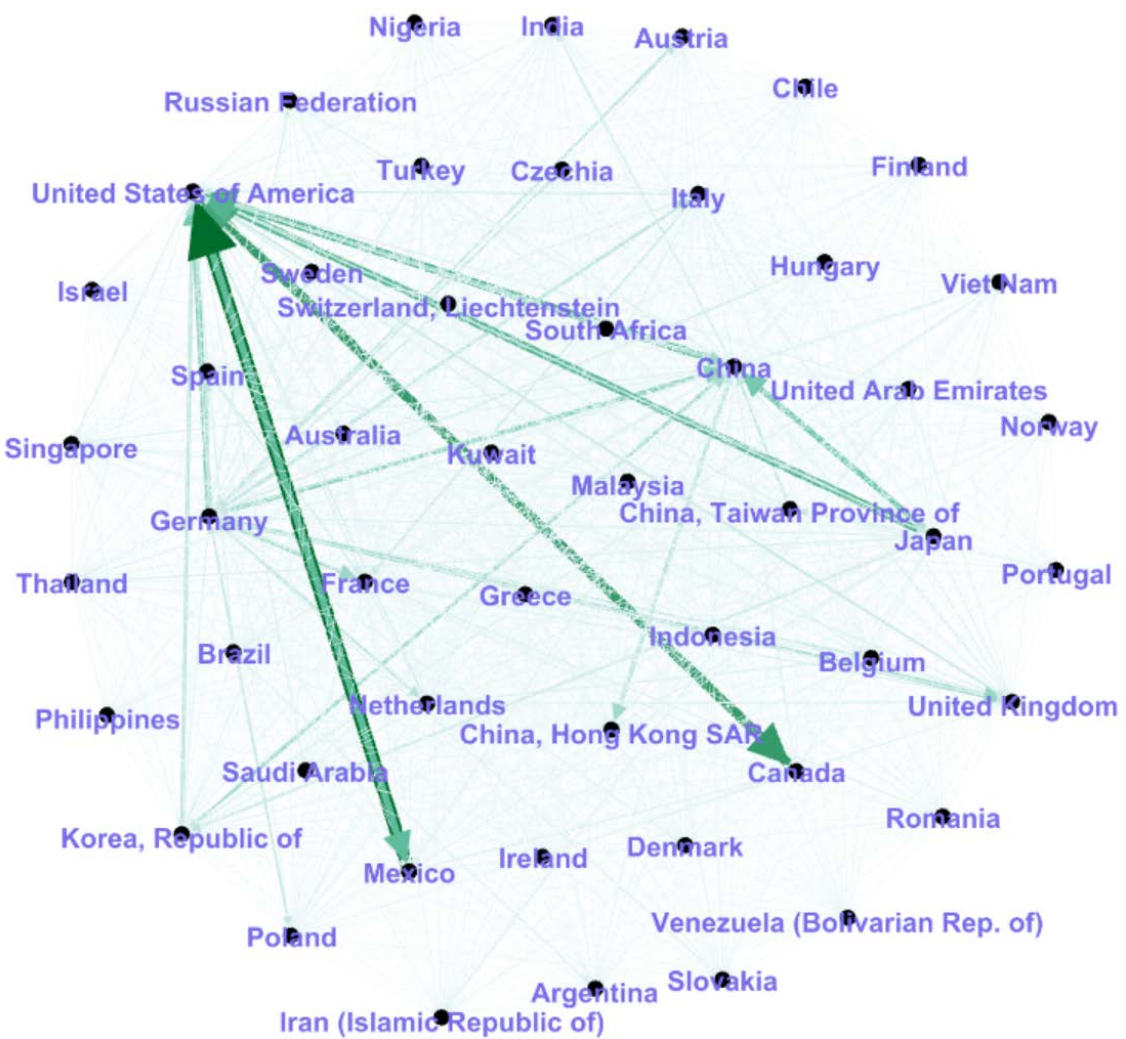

Figure 7. Medium-tech products trade network (short-term forecast). 


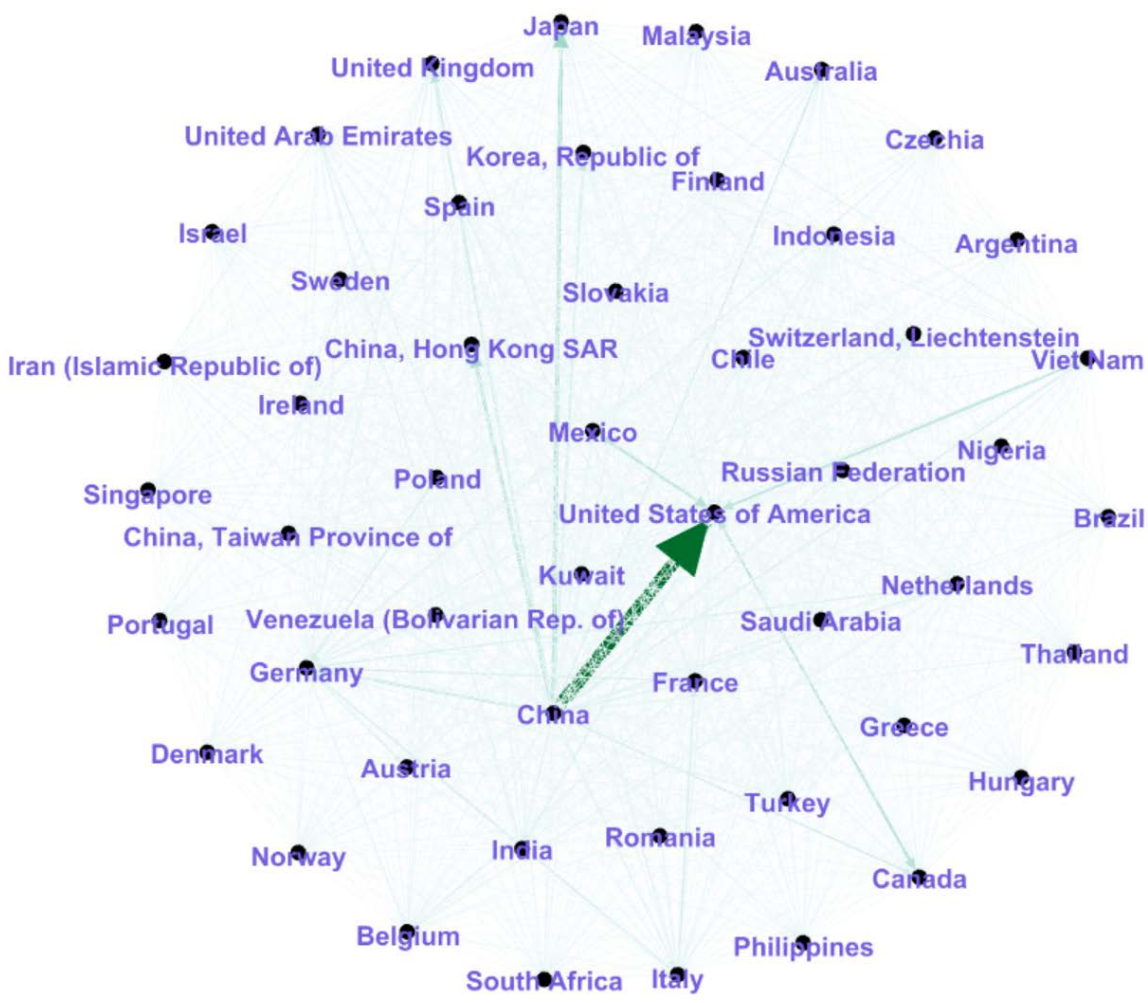

Figure 8. Low-tech products trade network (short-term forecast).

trend of high-tech products has continued to rise. Major trading importers include the United States, China, Hong Kong, Germany, and Japan. The major trade exporters include China, the United States, Germany, South Korea, and Taiwan. The trading trend of medium-tech products is more diversified. The United States is the largest importer, and its import volume is more than twice that of the second place China. Japan, Canada, Germany, and Mexico are also major trading countries of medium-tech products. The world's largest exporter of low-tech products is China. The largest importer of low-tech products is the United States. The United Kingdom, Japan, Hong Kong and Germany also maintain close trade relations with China in trade of low-tech products. From the perspective of overall manufacturing trade, China is currently the most competitive manufacturing country, but it is facing rising pressure on manufacturing costs. The United States and China will compete for the first place in the future. The US manufacturing industry will gradually recover its competitiveness due to its continuous productivity growth, exchange rate stability and energy cost advantages. The forecast data reveals that there will be three strong manufacturing regional clusters in the future which dominates the competitive landscape, namely North America (US, Canada and Mexico), European clusters (Germany and UK) and Asia Pacific clusters (China, Japan, Korea, Taiwan, and Hong Kong, China). From the perspective of products, global manufacturing trade will transform into high and medium tech manufacturing, creating a competitive advantage for the future of the economy. 


\section{Conclusion}

By constructing a global weighted network of manufacturing trade in 2001-2018, this paper analyzes its network degree distribution, network centrality, network degree correlation, network clustering and other characteristics, and carries out core-periphery analysis of the network. Based on the above research, we will make short-term predictions on the future trend of global manufacturing trade network development, and draw the following conclusions:

1) The average strength of global manufacturing trade in the past 18 years showed an overall upward trend, except for the impact of the global financial crisis in 2009. The trend of intensity distribution of products with different technical contents is similar. The current focus of the manufacturing trade system has shifted to enhanced trade flows, and very few countries occupy most of the trade flows in the network.

2) The focus of manufacturing trade in China is not limited to low-tech products, but to high-tech products, thus pushing the world's manufacturing center to move eastward. As the first importing country, the United States has not only relied heavily on high-tech products in recent years, but its demand for medium-tech products has soared in 2018 as well. Most of the high-central node trades have occurred in the past five years, indicating that countries have played a more active role in world manufacturing trade under the guidance of returning to manufacturing.

3) Trade networks have evolved over time, and researches have found that countries with low node degrees tend to develop trade relations with countries with high node degrees. Due to the proximity of geographical location and cultural similarity, small countries in local areas tend to develop manufacturing trade relations with hub countries in the region, and then form a regional economic cooperation organization with regional powers as the core.

4) The average clustering efficient of network in 50 countries or regions selected in this study is between 0.9465 and 0.9430 , which indicates that the relationship between networks is relatively stable, the accessibility and tightness are strong, the trade efficiency of commodity is high, and the network system tends to mature.

5) Nowadays, China and the United States are the core countries of the three categories of high, medium and low-grade products, and their status is stable and difficult to be replaced. The core countries of medium-tech products are distributed widely, and the core countries of high-tech products are mainly distributed in North America and Asia. Since 2018, high and medium-tech products have become the core countries' trade targets, indicating that the medium and high manufacturing industry has become the focus of global strategic competition among economies.

6) In the future, most of the trade flows of manufacturing trade are still in the hands of a few trading countries. China and the United States, two manufacturing powers, will face more intense competition. Global manufacturing trade will 
form a three-group situation: North American three countries (US, Canada, and Mexico), European clusters (Germany and the United Kingdom), and Asia-Pacific clusters (China, Japan, South Korea, Taiwan, China and Hong Kong, China). The global manufacturing industry will shift to the direction of high- and medium-tech products.

In this article, we combined time and space to explore the evolutions of global manufacturing trade by using multi-layer complex network. This study makes significant contributions to look for features of manufacturing trade networks and try to draw a blueprint for trade relations among economies in terms of technological classification. The insights gained from this study may be of assistance to identify the core trade cooperation groups. Also, we highlight the future pattern of manufacturing trade network by methods of HoltWinters. Through this study, we hope to provide a more systematic, comprehensive, dynamic and objective view of international trade in manufacturing, so as to promote the deepening and improvement of manufacturing trade in the future.

\section{Conflicts of Interest}

The authors declare no conflicts of interest regarding the publication of this paper.

\section{References}

Almog, A., Squartini, T., \& Garlaschelli, D. (2015). A GDP-Driven Model for the Binary and Weighted Structure of the International Trade Network. New Journal of Physics, 17, 169-174. https://doi.org/10.1088/1367-2630/17/1/013009

Barabasi, A. L., \& Albert, R. (1999). Emergence of Scaling in Random Networks. Science, 286, 509-512. https://doi.org/10.1126/science.286.5439.509

Barabasi, A. L., Jeong, H., Neda, Z., Ravasz, E., Schubert, A., \& Vicsek, T. (2002). Evolution of the Social Network of Scientific Collaborations. Physica A Statistical Mechanics and Its Applications, 311, 590-614. https://doi.org/10.1016/S0378-4371(02)00736-7

Boccaletti, S., Bianconi, G., Criado, R., del Genio, C. I., Gomez-Gardenes, J., Romance, M., Zanin, M. et al. (2014). The Structure and Dynamics of Multilayer Networks. Physics Reports-Review Section of Physics Letters, 544, 1-122. https://doi.org/10.1016/j.physrep.2014.07.001

De Domenico, M., Solè-Ribalta, A., Cozzo, E., Kivelä, M., Moreno, Y., Porter, M. A., Arenas, A. et al. (2014). Mathematical Formulation of Multi-Layer Networks. Physical Review X, 3, 4192-4195. https://doi.org/10.1103/PhysRevX.3.041022

Federico, B., Vincenzo, N., \& Vito, L. (2014). Structural Measures for Multiplex Networks. Physical Review E Statistical Nonlinear \& Soft Matter Physics, 89, Article ID: 032804. https://doi.org/10.1103/PhysRevE.89.032804

Gemmetto, V., Squartini, T., Picciolo, F., Ruzzenenti, F., \& Garlaschelli, D. (2015). Multiplexity and Multireciprocity in Directed Multiplexes. Physical Review E, 94, Article ID: 042316. https://doi.org/10.1103/PhysRevE.94.042316

Girvan, M., \& Newman, M. E. J. (2001). Community Structure in Social and Biological Networks. Proceedings of the National Academy of Sciences of the United States of America, 99, 7821-7826.

Halu, A., Mukherjee, S., \& Bianconi, G. (2015). Emergence of Overlap in Ensembles of 
Spatial Multiplexes and Statistical Mechanics of Spatial Interacting Network Ensembles. Physical Review E Statistical Nonlinear \& Soft Matter Physics, 89, 152-152. https://doi.org/10.1103/PhysRevE.89.012806

IMF (2019). Global Manufacturing Downturn, Rising Trade Barriers. Washington DC. https://www.imf.org/en/Publications/WEO/Issues/2019/03/28/world-economic-outloo k-april-2019

Kivelä, M., Arenas, A., Barthelemy, M., Gleeson, J. P., Moreno, Y., \& Porter, M. A. (2014). Multilayer Networks. SSRN Electronic Journal, 2, 261-268. https://doi.org/10.1093/comnet/cnu016

Lall, S. (2000). The Technological Structure and Performance of Developing Country Manufactured Exports, 1985-98. Oxford Development Studies, 28, 337-369. https://doi.org/10.1080/713688318

Lee, K. M., \& Goh, K. I. (2016). Strength of Weak Layers in Cascading Failures on Multiplex Networks: Case of the International Trade Network. Scientific Reports, 6, Article No. 26346. https://doi.org/10.1038/srep26346

Matteo, B., Giorgio, F., \& Diego, G. (2010). Multinetwork of International Trade: A Commodity-Specific Analysis. Physical Review E Statistical Nonlinear \& Soft Matter Physics, 81, Article ID: 046104. https://doi.org/10.1103/PhysRevE.81.046104

Roberts, B. (2004). A Gravity Study of the Proposed China-Asean Free Trade Area. The International Trade Journal, 18, 335-353. https://doi.org/10.1080/08853900490518208

Saracco, F., Clemente, R. D., Gabrielli, A., \& Squartini, T. (2015). Detecting the Bipartite World Trade Web Evolution across 2007: A Motifs-Based Analysis. Riccardo Di Clemente, 21, 88-94.

Shi, P., Zhang, J. et al. (2014). Hierarchicality of Trade Flow Networks Reveals Complexity of Products. PLoS ONE, 9, e98247. https://doi.org/10.1371/journal.pone.0098247

Solé-Ribalta, A., Domenico, M. D., Gómez, S., \& Arenas, A. (2016). Random Walk Centrality in Interconnected Multilayer Networks. Physica D Nonlinear Phenomena, 323-324, 73-79. https://doi.org/10.1016/j.physd.2016.01.002

Squartini, T., Fagiolo, G., \& Garlaschelli, D. (2011). Randomizing World Trade. I. A Binary Network Analysis. Physical Review E Statistical Nonlinear \& Soft Matter Physics, 84, Article ID: 046117. https://doi.org/10.1103/PhysRevE.84.046117

Tzekina, I., Danthi, K., \& Rockmore, D. N. (2008). Evolution of Community Structure in the World Trade Web. European Physical Journal B, 63, 541-545.

https://doi.org/10.1140/epjb/e2008-00181-2

Vázquez, A., Pastorsatorras, R., \& Vespignani, A. (2002). Large-Scale Topological and Dynamical Properties of the Internet. Physical Review E Statistical Nonlinear \& Soft Matter Physics, 65, Article ID: 066130. https://doi.org/10.1103/PhysRevE.65.066130

Watts, D. J., \& Strogatz, S. H. (1998). Collective Dynamics of "Small-World" Networks. Nature, 393, 440-442. https://doi.org/10.1038/30918

Zhang, X. H., Cui, H. Y., Zhu, J., Du, Y., Wang, Q., \& Shi, W. H. (2017). Measuring the Dissimilarity of Multiplex Networks: An Empirical Study of International Trade Networks. Physica A Statistical Mechanics and Its Applications, 467, 380-394.

https://doi.org/10.1016/j.physa.2016.10.024 


\section{Annex 1}

Table A1. Top 50 countries or regions in manufacturing trade (2001-2108).

\begin{tabular}{|c|c|c|c|c|c|}
\hline No. & countries or regions & $\%$ of trade & NO. & countries or regions & $\%$ of trade \\
\hline 1 & Argentina & $0.03 \%$ & 26 & Kuwait & $0.44 \%$ \\
\hline 2 & Australia & $1.29 \%$ & 27 & Malaysia & $1.39 \%$ \\
\hline 3 & Austria & $1.08 \%$ & 28 & Mexico & $2.26 \%$ \\
\hline 4 & Belgium & $3.05 \%$ & 29 & Netherlands & $3.99 \%$ \\
\hline 5 & Brazil & $1.25 \%$ & 30 & Nigeria & $0.46 \%$ \\
\hline 6 & Canada & $3.15 \%$ & 31 & Norway & $0.89 \%$ \\
\hline 7 & Chile & $0.42 \%$ & 32 & Philippines & $0.40 \%$ \\
\hline 8 & China & $10.04 \%$ & 33 & Venezuela (Bolivarian Rep. of) & $0.61 \%$ \\
\hline 9 & China, Hong Kong SAR & $3.09 \%$ & 34 & Poland & $1.04 \%$ \\
\hline 10 & China, Taiwan Province of & $1.93 \%$ & 35 & Portugal & $0.40 \%$ \\
\hline 11 & Czechia & $0.88 \%$ & 36 & Romania & $0.33 \%$ \\
\hline 12 & Denmark & $0.74 \%$ & 37 & Russian Federation & $2.44 \%$ \\
\hline 13 & Finland & $0.56 \%$ & 38 & Saudi Arabia & $1.65 \%$ \\
\hline 14 & France & $4.02 \%$ & 39 & Singapore & $2.33 \%$ \\
\hline 15 & Germany & $9.34 \%$ & 40 & Slovakia & $0.41 \%$ \\
\hline 16 & Greece & $0.21 \%$ & 41 & South Africa & $0.52 \%$ \\
\hline 17 & Hungary & $0.63 \%$ & 42 & Spain & $1.85 \%$ \\
\hline 18 & India & $1.42 \%$ & 43 & Sweden & $1.23 \%$ \\
\hline 19 & Indonesia & $0.85 \%$ & 44 & Switzerland, Liechtenstein & $1.60 \%$ \\
\hline 20 & Iran (Islamic Republic of) & $0.56 \%$ & 45 & Thailand & $1.28 \%$ \\
\hline 21 & Ireland & $0.92 \%$ & 46 & Turkey & $0.82 \%$ \\
\hline 22 & Israel & $0.42 \%$ & 47 & United Arab Emirates & $1.56 \%$ \\
\hline 23 & Italy & $3.54 \%$ & 48 & United Kingdom & $3.45 \%$ \\
\hline 24 & Japan & $5.43 \%$ & 49 & United States of America & $9.67 \%$ \\
\hline 25 & Korea, Republic of & $3.08 \%$ & 50 & Viet Nam & $0.61 \%$ \\
\hline
\end{tabular}

\section{Annex 2}

Table A2. Top 10 countries or regions in world manufacturing import (short-term forecast).

\begin{tabular}{cccccc}
\hline \multicolumn{2}{c}{ High-tech product ranking } & \multicolumn{2}{c}{ Medium-tech product ranking } & \multicolumn{2}{c}{ Low-tech product ranking } \\
\hline Countries or regions & Volume & Countries or regions & Volume & Countries or regions & Volume \\
\hline United States of America & $267,697,865.4$ & United States of America & $237,077,856.7$ & United States of America & $17,573,0221.3$ \\
China & $236,587,274.6$ & China & $119,870,560.7$ & Germany & $80,058,404.67$ \\
China, Hong Kong SAR & $159,141,429.7$ & Germany & $113,952,338.1$ & France & $48,887,101.11$ \\
Germany & $106,366,594.2$ & United Kingdom & $65,259,394.97$ & United Kingdom & $46,286,610.35$ \\
Japan & $63,130,724.61$ & France & $61,230,405.14$ & Japan & $42,494,961.75$ \\
\hline
\end{tabular}




\section{Continued}

\begin{tabular}{cccccr}
\hline Netherlands & $60,288,013.13$ & Canada & $56,303,628.33$ & China & $37,119,526.52$ \\
United Kingdom & $57,690,516.43$ & Mexico & $48,590,699.88$ & Netherlands & $34,715,176.62$ \\
France & $52,804,296$ & Italy & $43,144,779.2$ & China, Hong Kong SAR & $33,364,497.25$ \\
Korea, Republic of & $49,319,061.38$ & Netherlands & $40,157,230.41$ & Italy & $31,320,273.76$ \\
Singapore & $44,456,627.44$ & Japan & $40,039,531.56$ & Canada & $28,750,886.87$ \\
\hline
\end{tabular}

Table A3. Top 10 countries or regions in world manufacturing export (short-term forecast).

\begin{tabular}{cccccc}
\hline \multicolumn{2}{c}{ High-tech product ranking } & \multicolumn{2}{c}{ Medium-tech product ranking } & \multicolumn{2}{c}{ Low-tech product ranking } \\
\hline Countries or regions & Volume & Countries or regions & Volume & Countries or regions & Volume \\
\hline China & $403,465,758.2$ & Germany & $202,983,336$ & China & $297,313,103.8$ \\
United States of America & $145,409,985.5$ & China & $162,031,630$ & Germany & $74,089,406.54$ \\
Germany & $12,828,9087.8$ & United States of America & $155,211,320.1$ & United States of America & $53,340,033.25$ \\
Korea, Republic of & $93,662,773.63$ & Japan & $121,335,135.4$ & Italy & $51,272,346.85$ \\
China, Taiwan Province of & $92,684,257.22$ & Korea, Republic of & $65,070,376.41$ & Viet Nam & $35,011,295.85$ \\
China, Hong Kong SAR & $80,096,581.69$ & Mexico & $62,363,828.35$ & France & $32,649,872.38$ \\
Japan & $74,854,817.38$ & Italy & $58,465,672.64$ & India & $30,514,951.82$ \\
Singapore & $58,082,187.81$ & France & $55,417,532.46$ & Japan & $28,409,121.2$ \\
France & $55,591,772.75$ & United Kingdom & $47,973,418.18$ & Netherlands & $26,508,993.17$ \\
Malaysia & $51,864,036.66$ & Netherlands & $42,369,351.71$ & Korea, Republic of & $25,665,407.51$ \\
\hline
\end{tabular}

